\title{
High-Speed Time-Domain En Face Optical Coherence Tomography System Using KTN Optical Beam Deflector
}

\author{
Masato Ohmi1, Yusuke Shinya', Jun Miyazu², Seiji Toyoda ${ }^{2}$, Tadashi Sakamoto \\ ${ }^{1}$ Course of allied Health Science, Graduate school of Medicine, Osaka University, Yamadaoka, Suita, Osaka, Japan \\ ${ }^{2}$ NTT Device Innovation Center, NTT Corporation, Morinosato Wakamiya, Atsugi, Kanagawa, Japan \\ Email:ohmi@sahs.med.osaka-u.ac.jp
}

How to cite this paper: Ohmi, M., Shinya, Y., Miyazu, J., Toyoda, S. and Sakamoto, T. (2019) High-Speed Time-Domain En Face Optical Coherence Tomography System Using KTN Optical Beam Deflector. Optics and Photonics Journal, 9, 53-59. https://doi.org/10.4236/opj.2019.95006

Received: April 8, 2019

Accepted: May 7, 2019

Published: May 10, 2019

Copyright $\odot 2019$ by author(s) and Scientific Research Publishing Inc. This work is licensed under the Creative Commons Attribution International License (CC BY 4.0).

http://creativecommons.org/licenses/by/4.0/

\begin{abstract}
We developed high-speed time-domain (TD) en face optical coherence tomography (OCT) system using KTN optical beam deflector. The KTN optical beam deflector operates at a high repetition rate of $200 \mathrm{kHz}$ with a fairly large beam deflection angle. We proposed a high-speed en face OCT system that used a KTN optical deflector as the sample beam scanning. In the experiment, we obtained en face OCT images of human fingerprint with a frame rate of $800 \mathrm{fps}$, which is the fastest speed obtained by a TD-OCT imaging. Furthermore, a 3D-OCT image was also obtained at $0.2 \mathrm{~s}(=5$ volumes $/ \mathrm{s})$ by our imaging system.
\end{abstract}

\section{Keywords}

Optical Coherence Tomography, En Face OCT, KTN, Optical Beam Deflector

\section{Introduction}

Since the proposal of optical coherence tomography (OCT) [1], OCT has been developed intensively for clinical diagnoses in ophthalmology [2]. There are three types of data acquisition and image processing by OCT: Time domain (TD) OCT [1] [2]; Fourier domain (FD)/spectral domain (SD) OCT [3]; and Swept source (SS) OCT [4]. SS-OCT offers the highest speed for data acquisition due to the wavelength-tuning speed of a swept source. Recently, Microelectromechanical Systems (MEMS) vertical-cavity surface-emitting laser swept source provides sweeping speed of $60 \mathrm{kHz}$ to $1 \mathrm{MHz}$ [5]. The Fourier domain mode-locked laser (FDML) is considered to have the highest sweeping speed in the range of up to multi $\mathrm{MHz}$ [6]. Another imaging method for OCT is en face OCT, also 
known as optical coherence microscopy (OCM) [7], which performs perpendicular sectional imaging of a sample. Generally, a CCD camera is used as a detector that captures en face images of the depth direction of the sample to movement of a reference mirror, and acquires three-dimensional information. This imaging technique has a comparatively low $\mathrm{S} / \mathrm{N}$ ratio due to the low amount of radiation power used to illuminate a sample.

The $\mathrm{KTa}_{1-\mathrm{x}} \mathrm{Nb}_{\mathrm{x}} \mathrm{O}_{3}$ (KTN) crystal has a very large electro-optic (EO) effect, which changes its refractive index when a voltage is applied and bends the path of a light beam in a new direction. The deflection effect of the KTN is caused by a non-uniform electric field generated by injected carriers, and exhibits a fast response of up to several hundred $\mathrm{MHz}$ and a fairly large beam deflection angle [8] [9] [10]. Considering this performance, we proposed a high-speed en face OCT system that used a KTN optical deflector as the sample probe [11]. The OCT data acquisition rate of 400 frames/sec (fps) was obtained. In this paper, the highest frame rate of $800 \mathrm{fps}$ was obtained by use of heterodyne detection of the interference OCT signals. Furthermore, the 3D-OCT image of sweat gland was also obtained at $0.2 \mathrm{~s}$ by our imaging system.

\section{En Face 0CT System Configuration}

The high-speed en face OCT system configuration is shown in Figure 1. The light source of the Mach-Zehnder interferometer is a super-luminescent diode (SLD) light which is linearly polarized and has the spectral width of $56 \mathrm{~nm}$ around a central wavelength of $1.31 \mu \mathrm{m}$. The output power of the SLD light is 20 $\mathrm{mW}$. Light from the SLD is split by a $99 / 1$ coupler into one beam being transmitted down a sample arm and the other beam being transmitted down a reference arm. Acousto-optic (AO) modulator is placed on the reference arm in order to heterodyne detection of the interference signal. The modulation frequency is $40 \mathrm{MHz}$. Both beams are reflected by the reference mirror, and the sample beam is separated by optical circulators (CIR). Polarization controllers (PC) are used to adjust the polarization states of the beams in each arm. Light from the two arms interferes at a 50/50 coupler, whose outputs are detected by a balanced photo-detector. The detected interference signal is amplified and fed to an $\mathrm{AD}$

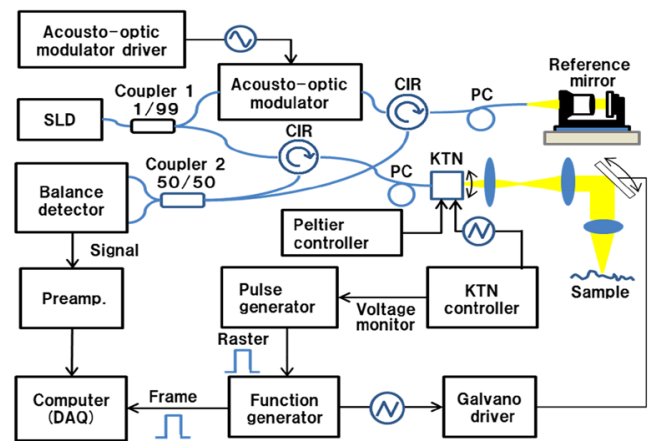

Figure 1. High-speed TD en face OCT system configuration. CIR: Circulator; PC: Polarization controller; DAQ: Data acquisition system. 


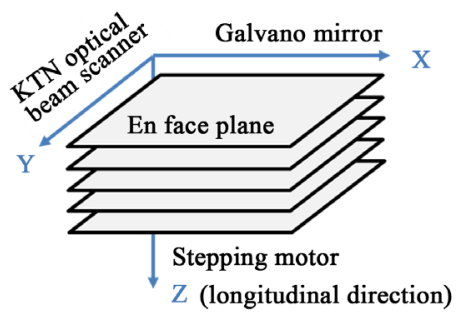

Figure 2. En face scanning for 3D-OCT dada.

converter. The sampling rate of the $\mathrm{AD}$ converter is $400 \mathrm{MHz}$ with 14-bit digitizer.

In the en face OCT imaging, the sample light is scanned along the $\mathrm{x}$-axis by a galvanometer mirror and along the y-axis by a KTN optical beam deflector, as shown in Figure 2. In our imaging system, the fast scanning was performed at $200 \mathrm{kHz}$ along the $\mathrm{y}$-axis, while the slow scanning was performed at $800 \mathrm{~Hz}$ along the $\mathrm{x}$-axis. For 3D-OCT imaging, the reference mirror was moved along the $\mathrm{z}$-axis by a stepping motor during the en face OCT imaging.

\section{Optical Arrangement of the Sample Arm}

The KTN deflector operates by exploiting electrons trapped in the KTN crystal. The electrons are injected and kept trapped by supplying a DC voltage to the KTN prior to the application of a high frequency AC voltage for scanning. We pre-charged the KTN crystal by applying $\pm 400 \mathrm{~V}$ DC for $10 \mathrm{~s}$, and then scanned the laser beam by applying a $\pm 300 \mathrm{~V}$ triangle wave voltage to the KTN deflector [8] [9]. This resulted in the beam deflected by an angle of $112 \mathrm{mrad}$. KTN simultaneously exhibited the characteristics of a cylindrical convex lens because of the trapped electrons [10]. We compensated for this convex lens effect with a cylindrical concave lens. Figure 3 shows the beam propagation and optical arrangement of the sample arm. To enlarge the input beam diameter of the focusing lens, we use $\times 3$ magnification relay optical system include $f_{1}$ and $f_{2}$ plano convex lenses.

In the experiment, the sample light is scanned along the $\mathrm{x}$-axis by a galvanometer mirror and along the y-axis by a KTN optical beam deflector. The lateral resolution of the en face OCT system can be measured by determining the smallest element of the test chart (USAF 1951) that can be clearly resolved. Figure 4 shows the test chart and the en face OCT image of the object. From the smallest resolvable element, a horizontal (x-direction) resolution of $13.9 \mu \mathrm{m}$ and a vertical (y-direction) resolution of $17.5 \mu \mathrm{m}$ were obtained, respectively. We also measured the sensitivity of the en face OCT system by inserting a neutral-density (ND) 4.6 filter into a sample arm. The signal-to-noise ratio (SNR) is given by the expression [12]

$$
\mathrm{SNR}=20 \log _{10}\left(\frac{\text { Signal amplitude }}{\text { Standard deviation of the noise }}\right)+P_{N D}[\mathrm{~dB}]
$$

where $P_{N D}$ is the signal power with the ND filter. A measured the SNR was $-67.31 \mathrm{~dB}$, as shown in Figure 5 . 


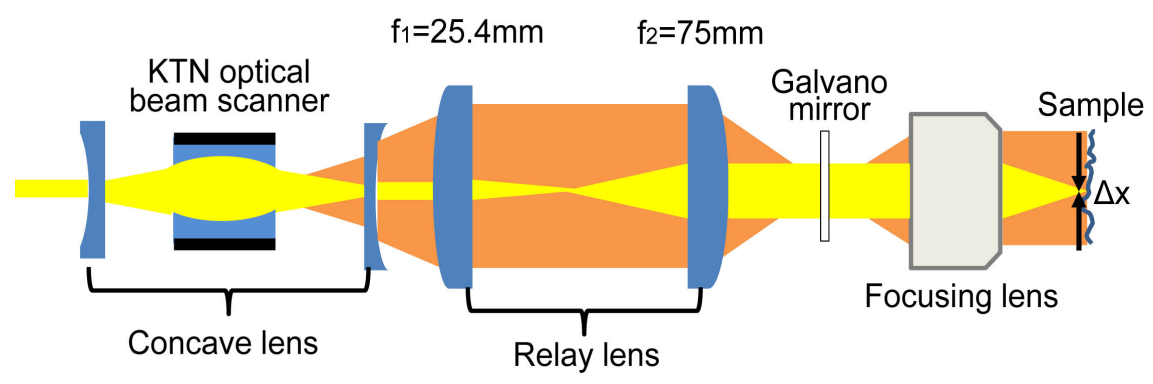

Figure 3. Beam propagation and optical arrangement of the sample arm.

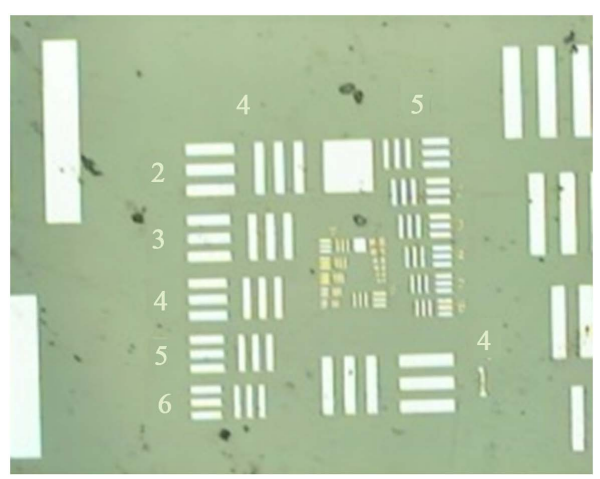

(a)

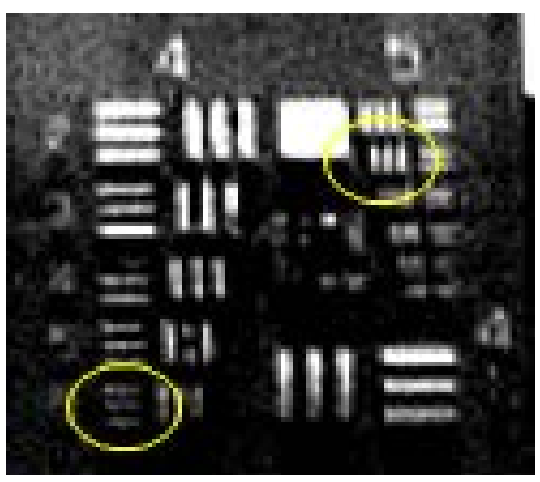

(b)

Figure 4. En face image of test chart (USAF 1951). (a) Photograph of test chart; (b) En face image of test chart.

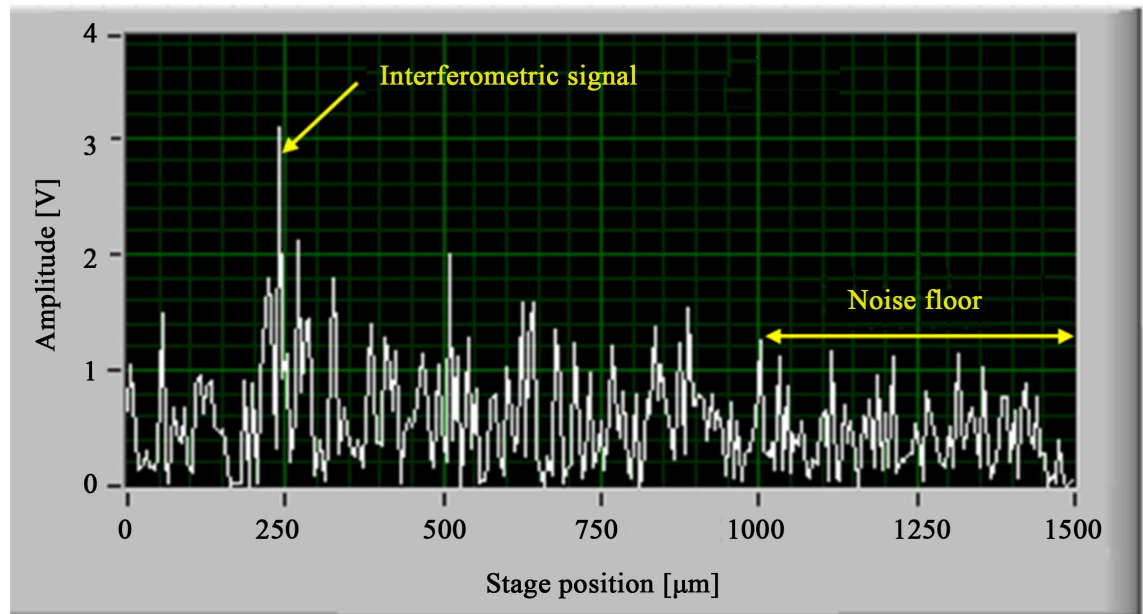

Figure 5. The measured signal-to-noise ratio (SNR) of the en face OCT system.

\section{High-Speed TD En Face OCT Imaging of Human Fingertip}

In the experiment, we acquired human fingerprint images using en face OCT system. The $250 \times 500$ pixel images covered a sample area of approximately 1.9 $\mathrm{mm} \times 1.9 \mathrm{~mm}(\mathrm{x}, \mathrm{y})$. The KTN beam deflector operated at $200 \mathrm{kHz}$ in the $y$-direction, whereas the galvanometer scans operated at $800 \mathrm{~Hz}$ in the $\mathrm{x}$-direction. Figure 6(a) shows the en face OCT image of the human fingerprint of $x-y$ plane. Figure 6(b) and Figure 6(c) show the OCT image of $y-z$ plane and 


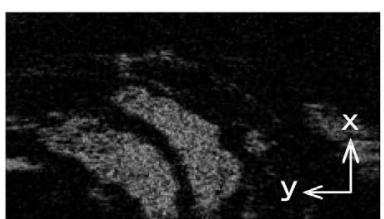

(a)

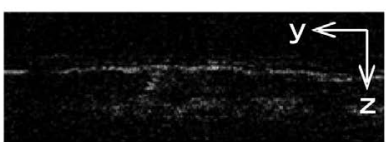

(b)

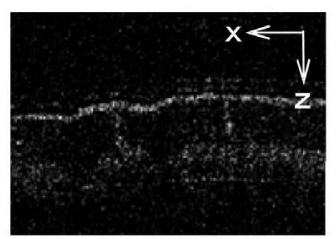

(c)

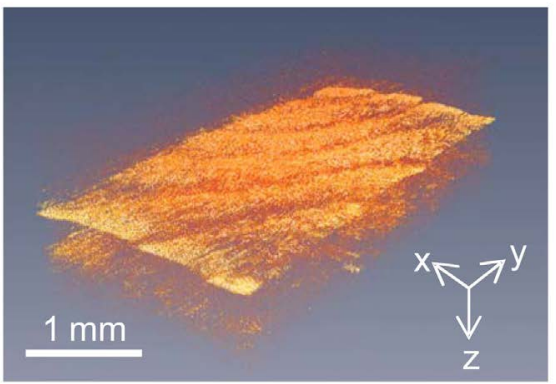

(d)

Figure 6. High-speed en face OCT images of human fingerprint. (a) En face OCT image of $x-y$ plane; (b) OCT image of the $y-z$ plane; (c) OCT image of $x-z$ plane; (d) Constructed 3D-OCT.

$\mathrm{x}-\mathrm{z}$ plane, respectively. The image acquisition rate was $800 \mathrm{fps}$, which is the highest speed obtained using en face OCT imaging and is comparable to the speed of SS-OCT systems. Furthermore, during 3D imaging, in addition to the operation of the galvanometer mirror and the KTN beam deflector of the sample arm, a reference mirror is moved for stacking en face OCT images. All the data from3D-OCT scanning was stored in the data acquisition board. As shown in Figure 6(d), the volume size of a 3D image was $250 \times 500 \times 160$ voxels $(x, y, z)$ with a $3 \mathrm{D}$ data acquisition rate of 5 volumes/s. The measurement ranges were $1.9 \mathrm{~mm} \times 1.9 \mathrm{~mm} \times 1.5 \mathrm{~mm}$. The surface profile of the human fingerprint is seen clearly, and sweat gland in the stratum corneum and epidermis are recognized.

\section{Conclusion}

In conclusion, we proposed and demonstrated high-speed TD en face OCT system using KTN optical beam deflector. In the imaging system, the KTN beam deflector operates at $200 \mathrm{kHz}$ in the $\mathrm{y}$-direction, while the galvanometer scans at $800 \mathrm{~Hz}$ in the $\mathrm{x}$-direction. This OCT imaging system essentially follows the TD method. The OCT data acquisition rate obtained is $800 \mathrm{fps}$, which is the highest frame-rate obtained by a TD en face OCT imaging. In the experiment, human fingerprints were presented as examples of en face and 3D-OCT images obtained at a volume rate of 5 volumes/s. Our efforts are directed at the development of a compact en face OCT system which conducts two dimensional scanning using only KTN beam deflectors. 


\section{Acknowledgements}

This research was supported by the AMED-SENTAN Program by Japan Agency for Medical Research and Development (AMED), for which we express our gratitude.

\section{Conflicts of Interest}

The authors declare no conflicts of interest regarding the publication of this paper.

\section{References}

[1] Huang, D., Swanson, E.A., Lin, C.P., Schuman, J.S., Stinson, W.G., Chang, W., Hee, M.R., Flotte, T., Gregory, K., Puliafito, C.A. and Fujimoto, J.G. (1991) Optical Coherence Tomography. Science, 254, 1178-1181. https://doi.org/10.1126/science.1957169

[2] Swanson, E.A., Izatt, J.A., Hee, M.R., Huang, D., Lin, C.P., Schuman, J.S., Puliafito, C.A. and Fujimoto, J.G. (1993) In Vivo Retinal Imaging by Optical Coherence Tomography. Optics Letters, 18, 1864-1866. https://doi.org/10.1364/OL.18.001864

[3] Nassif, N., Cense, B., Park, B.H., Yun, S.H., Chen, T.C., Bouma, B.E., Tearney, G.J. and De Boer, J.F. (2004) In Vivo Human Retinal Imaging by Ultrahigh-Speed Spectral Domain Optical Coherence Tomography. Optics Letters, 29, 480-482. https://doi.org/10.1364/OL.29.000480

[4] Yun, S.H., Tearney, G.J., De Boar, J.F., Iftimia, N. and Bouma, B.E. (2003) High-Speed Optical Frequency-Domain Imaging. Optics Express, 11, 2953-2963. https://doi.org/10.1364/OE.11.002953

[5] Potsaid, B., Jayaraman, V., Fujimoto, J.G., Jiang, J., Heim, P.J.S. and Cable, A.E. (2013) MEMS Tunable VCSEL Light Source for Ultrahigh Speed $60 \mathrm{kHz}-1 \mathrm{MHz}$ Axial Scan Rate and Long Range Centimeter Class OCT Imaging. Proceedings of SPIE Photonics West 2013, San Francisco, CA, United States, 2-7 February 2013, 82130M-1 to 82130M-8. https://doi.org/10.1117/12.911098

[6] Huber, R., Adler, D.C., Srinivasan, V.J. and Fujimoto, J.G. (2007) Fourier Domain Mode Locking at $1050 \mathrm{~nm}$ for Ultra-High-Speed Optical Coherence Tomography of the Human Retina at 236,000 Axial Scans Per Second. Optics Letters, 32, 2049-2051. https://doi.org/10.1364/OL.32.002049

[7] Beaurepaire, E., Boccara, A.C., Lebec, M., Blanchot, L. and Saint-Jalmes, H. (1998) Full-Field Optical Coherence Microscopy. Optics Letters, 23, 244-246. https://doi.org/10.1364/OL.23.000244

[8] Nakamura, K., Miyazu, J., Sasaura, M. and Fujiura, K. (2006) Wide-Angle, Low-Voltage Electro-Optic Beam Deflection Based on Space-Charge-Controlled Mode of Electrical Conduction in $\mathrm{KTa}_{1-\mathrm{x}} \mathrm{Nb}_{\mathrm{x}} \mathrm{O}_{3}$. Applied Physics Letters, 89, Article ID: 131115 . https://doi.org/10.1063/1.2357335

[9] Miyazu, J., Imai, T., Toyoda, S., Sasaura, M., Yagi, S., Kato, K., Sasaki, Y. and Fujiura, K. (2011) New Beam Scanning Model for High-Speed Operation Using $\mathrm{KTa}_{1-\mathrm{x}} \mathrm{Nb}_{\mathrm{x}} \mathrm{O}_{3}$ Crystals. Applied Physics Express, 4, Article ID: 111501. https://doi.org/10.1143/APEX.4.111501

[10] Imai, T., Miyazu, J. and Kobayashi, J. (2014) Charge Distributions in $\mathrm{KTa}_{1-\mathrm{x}} \mathrm{Nb}_{\mathrm{x}} \mathrm{O}_{3}$ Optical Beam Deflectors Formed by Voltage Application. Optics Express, 22, 14114-14126. https://doi.org/10.1364/OE.22.014114 
[11] Ohmi, M., Fukuda, A., Miyazu, J., Ueno, M., Toyoda, S. and Kobayashi, J. (2015) Development of Novel High-Speed En Face Optical Coherence Tomography System Using KTN Optical Beam Deflector. Applied Physics Express, 4, Article ID: 027001. https://doi.org/10.7567/APEX.8.027001

[12] Choma, M.A., Sarunic, M.V., Yang, C. and Izatt, J.A. (2003) Sensitivity Advantage of Swept Source and Fourier Domain Optical Coherence Tomography. Optics EXpress, 11, 2183-2189. https://doi.org/10.1364/OE.11.002183 